\title{
SISTEMA DE MEJORA CONTINUA PARA EL PROCESO CRM DE UNA EMPRESA DE SERVICIOS, DE TIJUANA, B.C., MÉXICO
}

\author{
Isaac Cruz Estrada*
}

\begin{abstract}
Cruz-Estrada I. Sistema de mejora continua para el proceso $\mathrm{crm}$ de una empresa de servicios, de Tijuana, B.C., México. Hitos de Ciencias Económico Administrativas 2015;21 (59): 33-42
\end{abstract}

RESUMEN

Objetivo: Implementación de un sistema de mejora continua (SMC) para una empresa de servicios de la ciudad de Tijuana, B.C., México, con la finalidad de mejorar el desempeño del proceso de gestión de relaciones con el cliente (CRM) implementado en la organización bajo estudio.

Material y método: El sistema se integró a una empresa de servicios de la ciudad de Tijuana, B.C., México, de este modo el trabajo parte de una revisión de sistemas de medición del desempeño, además de fundamentar los elementos contemplados en el CRM de la compañía, así mismo se señalan las herramientas que evalúan al CRM al SMC como elementos del proceso implantado.

Resultados: Como resultado se presentan las etapas y/o proceso a seguir por el SMC, buscando incrementar el desempeño del proceso CRM, de este modo se señalan los instrumentos a utilizar, los cuales actualmente forman parte de la implementación del programa en la organización bajo estudio.

Conclusiones: EI SMC desarrollado para el proceso CRM en la organización analizada requiere de involucrar a toda la empresa, ya que las estrategias resultantes deben ser construidas a partir de las áreas de oportunidad descubiertas y las aportaciones cualitativas

\author{
Cruz-Estrada I. Sistema de mejora continua para el \\ proceso crm de una empresa de servicios, de Tijuana, \\ B.C., México. Hitos de Ciencias Económico \\ Administrativas 2015;21 (59): 33-42
}

\section{ABSTRACT}

Objective: Implementing a continuous improvement system (SMC) for a service of the city of Tijuana, BC, Mexico, in order to improve the performance of process Customer Relationship Management (CRM) developed the organization under study.

Material and method: The system is integrated into a service company of the city of Tijuana, BC, Mexico, thus the work of a review of performance measurement systems, in addition to substantiate the items referred to in the CRM company, also tools that evaluate the CRM to SCM as elements of the process are indicated implemented.

Results: As a result the steps and / or process are presented below by the SMC, looking to increase the performance of the CRM process, thus the instruments used are indicated, which are currently part of program implementation in the organization under study.

Conclusions: He SMC developed for the CRM process in the analyzed organization requires involve the whole company because the resulting strategies must be constructed from the areas of opportunity discovery and qualitative input from the team that maintains contact with the market. The experience developed in the implanted cyclical process allows continuously innovate

* Maestro. Facultad de Turismo y Mercadotecnia. Universidad Autónoma de Baja California.

Fecha de recibido: 4 de febrero de 2015. Fecha de aceptación: 26 de marzo de 2015. 
del equipo de trabajo que mantiene contacto con el mercado. La experiencia desarrollada en el proceso cíclico implantado permite innovar de manera continua las formas en que la organización hace frente a las necesidades del entorno, siempre buscando cumplir con las expectativas y exigencias del mercado. the ways in which the organization addresses the needs of the environment, always seeking to meet the expectations and demands of the market.
Palabras clave:SMC. Evaluación del desempeño. CRM. Satisfacción del cliente.
Key words: SMC. Performance evaluation. CRM. Customer satisfaction.

DIRECCIÓN PARA RECIBIR CORRESPONDENCIA: Correo electrónico: icruz@uabc.edu.mx

$\mathbf{E}$

$\mathrm{n}$ el entorno actual de los negocios se vive con mayor intensidad la necesidad de hacer uso de la evaluación del desempeño individual y de los procesos en las organizaciones, dada la oportunidad de cumplir en el tiempo estipulado las metas propuestas. Para Arriagada (2002), la medición del desempeño, es un esfuerzo sistemático aplicado en las compañías para evaluar su gestión orientada al cumplimiento de su misión y visión, a partir de la optimación de los procesos que la componen, siendo que cada empresa vive un caso particular que debe ser abordado, analizando las áreas de oportunidad que se necesitan trabajar, para corregir los detalles encontrados durante las evaluaciones.

En un estudio desarrollado por Santana-Robles \& Granillo-Macías (2012) señalan a la medición del desempeño como fundamental para el ambiente de los negocios, ya que a través de esta se puede conocer, evaluar, controlar y corregir los alcances de los objetivos estratégicos de una empresa en particular. De este modo es transcendental el establecimiento de métricas que permitan monitorear el estado presente y futuro de los planes de acción desarrollados en la compañía, para entonces obtener los datos que traducen el nivel de los resultados alcanzados, los cuales descubren brechas que requieren ser atendidas.

En este sentido Guerra-López (2007) puntualiza, que el monitoreo del desempeño es uno de los elementos claves para llevar a cabo la mejora continua en los sistemas de la organización. Así mismo el Sistema de Mejora Continua (SMC) propuesto para la empresa bajo estudio permite ser el medio que integra y evalúa los elementos considerados para el éxito sistema CRM, definiendo y estipulando con claridad los aspectos que apoyaran a mejorar su desempeño y la intervención requerida que habrá de contribuir al desarrollo del negocio.

\section{REVISIÓN DE LITERATURA}

Los sistemas de evaluación del desempeño, se han convertido en uno de los programas claves para encontrar las deficiencias que limitan los resultados individuales y de los elementos en conjunto que conforman la organización, los cuales han estado condicionando el alcance de las metas establecidas. De acuerdo con Guerra-López (2007) estos se convierten en instrumentos, que contribuyen a la toma de decisiones objetiva que se espera tengan un impacto positivo en las metas establecidas, determinando además la eficiencia de los recursos establecidos que han sido recabados en los sistemas utilizados.

De este modo se puede mencionar al sistema de medición de desempeño; Análisis, Diseño, Desarrollo, Implementación y Evaluación (Analysis, Desing, Development, Implementation, and Evaluation ADDIE), utilizado en un estudio desarrollado por Loor (2013), como un proceso sistemático e interactivo, en donde los resultados de la evaluación formativa de cada fase, conduce al diseño de regreso a cualquiera de las etapas previas. El resultado final de cada punto, es el producto de inicio de la siguiente fase. En la figura 1 se expone la representación del modelo al cual hace referencia el autor. 


\section{Figura 1}

\section{Modelo ADDIE}

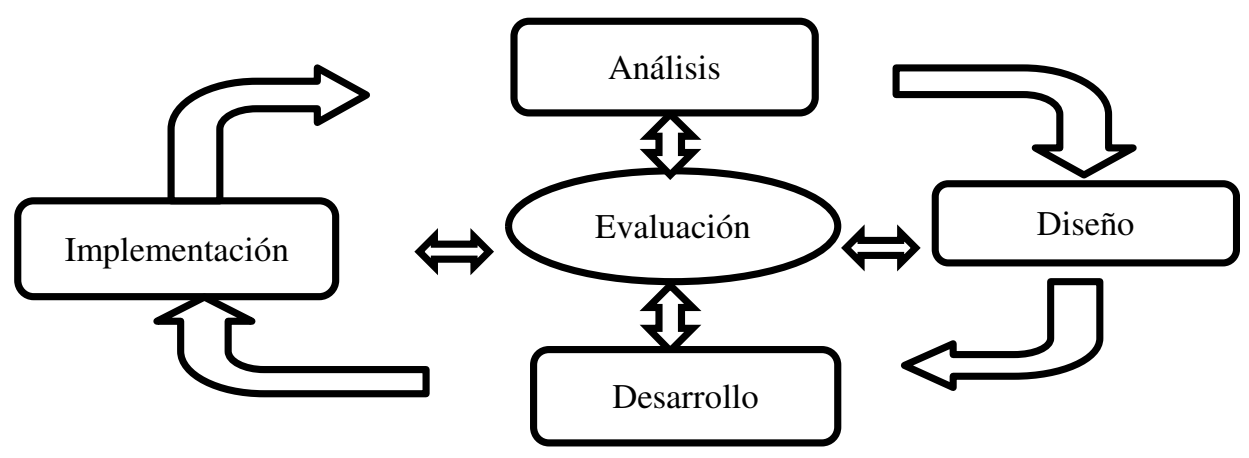

Fuente: Elaboración propia a partir de MunPoz \& Gonzaìlez (2009).

De acuerdo con Muñoz \& González (2009) la fase de análisis es la base de las otras etapas, en ella se deben evaluar las necesidades del entorno, para definir el problema, identificar la causa de la situación estudiada y buscar de esta manera posibles soluciones. Se pueden incluir en estas fases técnicas específicas de investigación, lo siguiente: análisis de necesidades, análisis de contexto y análisis de tareas. Así mismo en el ciclo que corresponde al diseño, implica la utilización de los resultados arrojados en la etapa de análisis, con el objetivo de planificar una estrategia para el desarrollo de la instrucción. Durante este periodo, es necesario especificar la forma en cómo se alcanzarán las metas determinadas en la fase de análisis y de este modo ampliar los fundamentos de la misma.

En este sentido, en la etapa de desarrollo el mismo autor describe que, el propósito de esta fase es generar las unidades, módulos y los materiales para el desarrollo de las mismas, así mismo en la etapa de implantación tiene como objetivo hacer funcionar el programa establecido de forma eficiente y efectiva. La ejecución del programa formativo, puede referirse a la ejecución del prototipo, a una implementación piloto o bien a una implementación total del proyecto desarrollado. Para la evaluación, pese a presentarse como la quinta etapa del modelo del diseño instruccional, es un componente integral de cada una de las cuatro fases anteriores. De este modo al conducir cada fase del diseño instruccional, los procedimientos y actividades pueden ser evaluados, para asegurar que se realicen de forma eficiente y eficaz para asegurar los resultados esperados.
Por otra parte, con base en Kaplan y Norton (2004) para construir un sistema de medición que describa la estrategia, aseguran que se requiere de un modelo general que sirva de base. El Balanced Scorecard, es una metodología para lograr trasladar la visión a la acción, ayuda a la organización a implementar un plan estratégico, a conseguir resultados innovadores rápidamente mediante la ejecución de un conjunto de estrategias y actividades, de este modo alcanzar las metas planteadas es más sencillo y fácil, para Vogel (2012) los indicadores deben mostrar «cómo nos vemos a nosotros mismos» y "cómo nos ven los demás» $y$, además, permitir las comparaciones en el tiempo y con otros indicadores y, en especial, que estén vinculados, esto quiere decir, que existe una relación causa-efecto. En la figura 2, se exponen las perspectivas que se deben considerar al construir el cuadro de mando integral.

Así mismo, Amo (2011) añade que no obstante, pudiera ser que dependiendo de la estrategia definida y de los grupos de interés del sector y las circunstancias, se hiciera necesario incluir una perspectiva adicional, dado que en ocasiones las organizaciones tienen un componente social, lo cual las lleva a adquirir un compromiso con su entorno, de esta manera, se pudiera incorporar otro elemento que contemple las acciones necesarias, para el cumplimento de las metas que deben estar establecidas en este nivel estratégico.

De acuerdo con Bernardez (2009), otra manera de evaluar el nivel de desempeño de los elementos que conforman un sistema organizacional, es a través del método creado por Kaoru Ishikawa en 1949, el cual permite analizar las causas de los problemas de 
Figura 2

Perspectivas del cuadro de mando

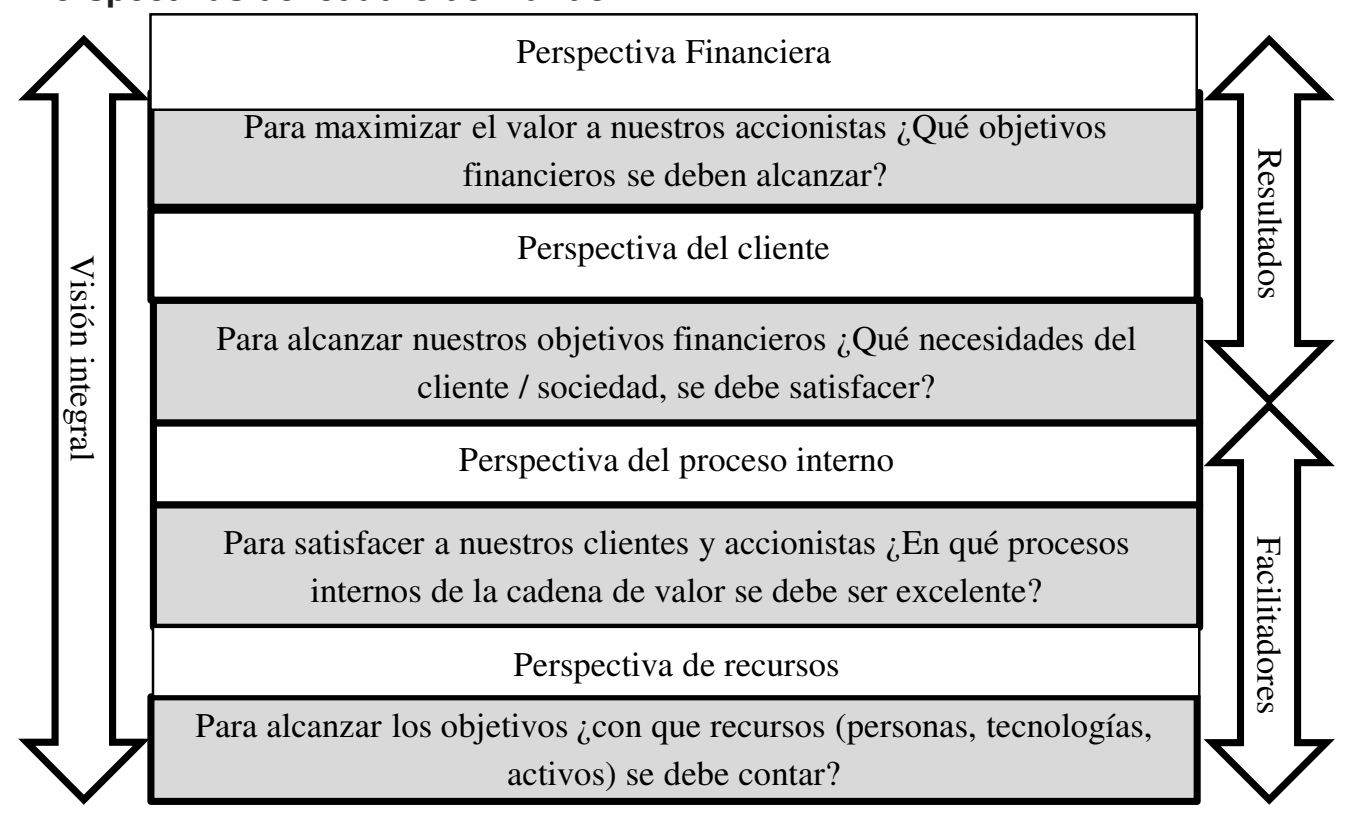

Fuente: Elaboración propia a partir de Amo (2011).

performance y calidad. En la cabeza del sistema, se debe colocar cada una de las brechas de performance detectadas y que requieren ser resueltas, y en las ramas de este esquema se colocan las causas posibles, agrupadas en factores asociados a las situaciones señaladas.

De acuerdo con el autor, la representación de los elementos considerados en la figura 3 , las causas de las brechas entre el performance actual y el deseado no solo operan linealmente, la acción de causa-efecto lo hace también de forma cíclica, en donde se evalúan los avances y estados alcanzados que ponen al descubierto las nuevas deficiencias encontradas que tendrán que irse mejorando, siendo que una vez cumplidas las metas, estás a su vez traerán nuevos retos que vienen acompañados de nuevas causales, que necesitan ser intervenidas para mejorar el desempeño de las situaciones descubiertas.

\section{ESTUDIOS SOBRE CRM}

En un estudio desarrollado por Cabanelas y Moreira (2009) realizado a organizaciones con la característica de alta rentabilidad (AR) en la región de Galicia, España, en la que como objetivo argumentan la importancia que le dan este tipo de organizaciones a la gestión de relaciones con el cliente, exponen que las empresas
AR asumen la gestión con los clientes como una apuesta estratégica, que incluye una filosofía de actuación ejecutada, principalmente, a través de relaciones informales explotadas por sus recursos humanos. La forma en que la construyen varía en función del tamaño o del sector, aunque dentro de un patrón definido.

La orientación de la estrategia a la que se refiere el autor en el anterior párrafo se basa en: mejorar las especificaciones propias del producto ofrecido (características tangibles); esforzarse en dar respuestas personalizadas a cada cliente (especialmente en las de menor tamaño) y potenciar la fidelización, como principio de eficiencia operativa (industria) o como propuesta de valor (empresas de servicios), siempre dentro de un contexto en el que la imagen de la empresa interviene como un factor clave en las relaciones con el mercado.

Por otra parte, Valenzuela y Torres (2008) presentan un estudio realizado en España dentro del sector turístico, en las agencias de viajes de ese país. Planteando que las empresas toman mejores decisiones estratégicas de marketing basadas en la gestión de su cartera de clientes, cuanto mayor sea el grado de orientación al valor que le den al mismo; de este modo aumentará la 
tasa de retención de los que son rentables; así como la optimización de sus costos de adquisición y en este sentido mejorarán los ingresos generados por los clientes durante su tiempo de vida.

A partir del estudio empírico mencionado en al anterior párrafo, se destaca que efectivamente las empresas que toman decisiones estratégicas de marketing orientadas al valor de su cartera de clientes mejoran su tasa de retención de estos y los ingresos generados durante su tiempo de vida, dado que al conseguir que los clientes rentables permanezcan leales a la organización, se logra que éstos continúen produciendo el ingreso esperado o bien evolucionen en sus compras lo cual se traduce en un mayor beneficio para el negocio.

De este modo, Garrido (2008) presenta un estudio empírico, en la que la población que interviene son los establecimientos hoteleros de tres a cinco estrellas situados en España. La investigación presenta como objetivos analizar el concepto de CRM; examinar su estado de la investigación; estudiar y analizar las variables que inciden su éxito en las estrategias utilizadas; examinar empíricamente el grado de desarrollo e implementación en el sector hotelero español, extrayendo conclusiones generalizables para otros sectores; y diseñar empíricamente un modelo de éxito.

Como resultado del trabajo desarrollado por Garrido (2008) la estimación empírica le permitió contrastar las distintas hipótesis de su investigación. Los resultados demostraron que las variables organizativas están relacionadas positivamente con los resultados del CRM, ejerciendo un impacto positivo y directo sobre los mismos. El resto de variables (tecnología, capacidades de adquisición y aplicación de conocimiento, capacidades de difusión y transmisión del conocimiento y orientación al cliente) no mostraron un impacto directo sobre los resultados del CRM, sino que ejercieron un impacto indirecto, mediado por las variables organizativas. Asimismo, la experiencia en la incorporación del proceso también impacta en los resultados, de manera que a medida que se incrementan los años de utilización de la estrategia, las empresas desarrollaban el aprendizaje organizativo necesario, incrementando así los resultados de la misma.
Así mismo, en un trabajo realizado por Guijarro (2009) el cual tiene como objetivo central de la investigación profundizar el impacto que pueda tener sobre el cliente la implantación de una estrategia cliente - céntrica en la agencia de viajes, aportando una visión teórica que respalde las primeras interpretaciones de esta nueva filosofía de gestión, sobre el futuro desarrollo del mundo empresarial. Los resultados aseveran la necesidad de considerar el uso de herramientas de Internet las cuales en la actualidad ha redefinido las reglas de los negocios tradicionales al crear un canal alternativo de venta e interacción con el cliente. Algo fundamental a desarrollar es la confianza en sus clientes, está aumentará cuando se perciba el buen hacer por parte de la empresa, y su preocupación por satisfacer sus necesidades.

Así mismo, Abad (2009) llevó a cabo un estudio en México para un hotel de cuatro estrellas, teniendo como objetivo diseñar un modelo CRM, que permita elevar la competitividad de la organización, detectando las áreas de mejora y de este modo perfeccionar la relación con los clientes. Entre los principales hallazgos se destaca la desventaja tecnológica y las posiciones estáticas, para comprender que las necesidades de la demanda constituyen las principales barreras del entendimiento del cliente reflejadas en la poca capacidad de servicios de los hoteles de cuatro estrellas. La gestión de servicios en los hoteles de cuatro estrellas deben de integrar una serie de funciones dirigidas hacia el conocimiento de las acciones del CRM, de tal modo que se fortalezca el vínculo de conocimiento entre la relación gerencia-cliente y prestador de servicios-cliente necesarios, para integrar nuevas relaciones en los servicios que doten a los sistemas de los hoteles de niveles mayores de competencia.

\section{MATERIAL Y MÉTODO}

El sistema de medición de desempeño, es resultado de la necesidad de dar seguimiento al modelo CRM implantado en la organización bajo estudio. Siendo que es necesario monitorear y estar adecuando las áreas de oportunidad encontradas, las cuales permitan adaptar a través del tiempo un sistema CRM flexible, capaz de incorporar situaciones que el entorno requiera y que estas sean adaptadas de acuerdo a las nuevas situaciones que enfrente la compañía. 
De este modo en la Tabla 1 se expone la comparativa de los tres sistemas de evaluación del desempeño descritos anteriormente en la revisión literaria, que son la base para el establecimiento del sistema.
Por otra parte en la Tabla 2 se muestran los elementos que configuran las variables que son parte de la estrategia CRM, los cuales son la base en la que se desarrolla el modelo implementado en la organización bajo estudio.

Tabla 1

Comparativa de los sistemas de evaluación considerados

\begin{tabular}{|c|c|c|}
\hline & Ventajas & Desventajas \\
\hline Modelo ADDIE & $\begin{array}{l}\text { - Es modelo genérico y sistémico } \\
\text { utilizado para diferentes contextos: } \\
\text { educativo, comercial, } \\
\text { gubernamental } \\
\text { - Es un modelo sencillo de } \\
\text { implementar } \\
\text { - Cada etapa es el resultado de la } \\
\text { etapa anterior }\end{array}$ & $\begin{array}{l}\text { - Al ser cada etapa dependiente de la } \\
\text { etapa anterior, el modelo puede } \\
\text { tornarse lento, cuando una etapa no } \\
\text { funcione correctamente, puede crear } \\
\text { demoras en la implementación }\end{array}$ \\
\hline $\begin{array}{l}\text { Cuadro de Mando o } \\
\text { Balanced Scorecard }\end{array}$ & $\begin{array}{l}\text { - Se tiene conciencia sobre la } \\
\text { estrategia en cada elemento del } \\
\text { sistema } \\
\text { - Se crea una ruta de objetivos } \\
\text { estratégicos } \\
\text { - Genera la cultura de la medición }\end{array}$ & $\begin{array}{l}\text { - Es necesario un análisis profundo } \\
\text { para que los objetivos estratégicos, } \\
\text { sean claramente medibles } \\
\text { - Si no es comunicado con eficiencia, } \\
\text { puede ocasionar una mala } \\
\text { interpretación de la estrategia por } \\
\text { parte de los colaboradores }\end{array}$ \\
\hline $\begin{array}{l}\text { Modelo Causa Efecto } \\
\text { de Ishikawa }\end{array}$ & $\begin{array}{l}\text { - Exige considerar una gran cantidad } \\
\text { de elementos asociados al } \\
\text { problema } \\
\text { - Se concentra en el proceso y no en } \\
\text { el producto }\end{array}$ & $\begin{array}{l}\text { - En un solo elemento se pueden } \\
\text { identificar demasiadas causas } \\
\text { potenciales } \\
\text { - Puede llegar a concentrarse en } \\
\text { pequeños detalles del proceso }\end{array}$ \\
\hline
\end{tabular}

Fuente: Elaboración propia a partir de Muñoz \& González (2009); Amo (2011); Bernárdez (2009).

Tabla 2

Elementos considerados en la estrategia CRM

\begin{tabular}{lccccc}
\hline & $\begin{array}{c}\text { Orientación } \\
\text { al cliente }\end{array}$ & $\begin{array}{c}\text { Gestión de la } \\
\text { información }\end{array}$ & $\begin{array}{c}\text { Gestión del } \\
\text { conocimiento }\end{array}$ & Tecnología & Innovación \\
\hline Cabanelas y & $\mathrm{X}$ & & $\mathrm{X}$ & $\mathrm{X}$ & $\mathrm{X}$ \\
Moreira (2009) & $\mathrm{X}$ & $\mathrm{X}$ & $\mathrm{X}$ & $\mathrm{X}$ & $\mathrm{X}$ \\
Garrido (2008) & $\mathrm{X}$ & $\mathrm{X}$ & $\mathrm{X}$ & $\mathrm{X}$ & $\mathrm{X}$ \\
Abad (2009) & $\mathrm{X}$ & $\mathrm{X}$ & & $\mathrm{X}$ \\
Valenzuela y & $\mathrm{X}$ & $\mathrm{X}$ & $\mathrm{X}$ & $\mathrm{X}$ & $\mathrm{X}$ \\
Torres (2008) & & $\mathrm{X}$ &
\end{tabular}

Fuente: Elaboración propia a partir de Cabanelas y Moreira (2009); Garrido (2008); Abad (2009); Valenzuela y Torres (2008); Guijarro (2009). 
Los elementos expuestos en la tabla 2, son considerados en los instrumentos del sistema de evaluación del desempeño, para el cual se tendrán que tomar las acciones necesarias, con la intención de ajustar las deficiencias encontradas que inhiben la eficiencia del proceso CRM. Así mismo, se incrementarán los resultados del sistema de marketing de la compañía, el cual pretende contribuir en el aumento de la competitividad del negocio por mediante la satisfacción de los clientes en el mercado de venta de café en la ciudad de Tijuana, Baja California.

\section{RESULTADOS}

En la figura 3, se muestra el SMC que debe seguir el proceso CRM en la organización bajo estudio, el cual se implementa con la intención de mejorar la satisfacción de los clientes, instituyéndose a este como su indicador principal. La visión MEGA de la compañía requiere de este proceso, para estar en la búsqueda de la creación de valor continuamente, mejorando constantemente los elementos que forman parte del mismo, ya que el cambio de las expectativas de los consumidores debe ser cuidado a partir del compromiso asumido de la compañía con su entorno con ética y responsabilidad.
El SMC propuesto, contempla tres instrumentos de medición, el primero dirigido al gerente o al coordinador de marketing de la organización para evaluar el desempeño y resultados del CRM en la organización; el segundo aplicado a los colaboradores en la creación de valor del producto y servicio entregado a los consumidores, evaluando la orientación al cliente y la gestión del conocimiento, los cuales son capacidades que debe tener el personal de la compañía; el tercer instrumento es aplicado a los clientes para evaluar la satisfacción del servicio y producto adquirido.

Estas técnicas otorgaran resultados que serán valorados de acuerdo a los parámetros ideales resueltos. De este modo, si existen limitantes para el desempeño del proceso se procede a corregir las incidencias encontradas, así mismo en ambos sentidos se buscará mejorar las formas y acciones empleadas para continuar con la mejora continua del CRM que lleva a satisfacer las necesidades de los clientes. En ese sentido, es fundamental el compromiso de todos los participantes y conocer en su totalidad el SMC propuesto, ya que las etapas se estarán alimentando de datos o elementos desarrollados en anteriores fases.

\section{Figura 3}

\section{SMC del proceso CRM de la organización}

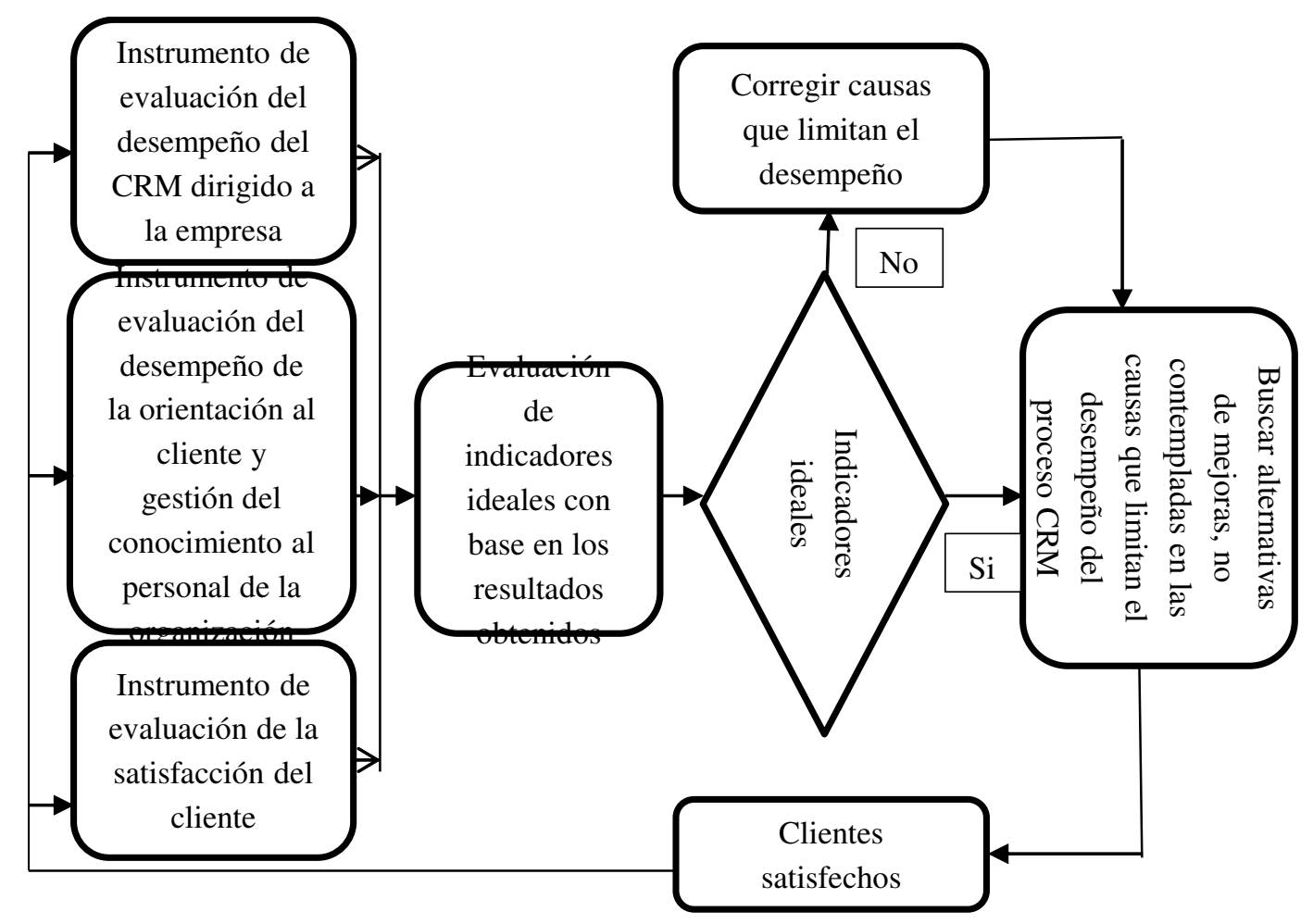




\section{DISCUSIÓN}

Las etapas consideradas para el SMC propuesto para la incrementar el desempeño del proceso CRM, tienen como punto principal la satisfacción del cliente, de este modo el ciclo establecido el cual lleva a la mejora continua del proceso, traerá como consecuencia el incremento del valor del producto y servicio ofrecido por la organización. Es necesario trabajar sobre las incidencias encontradas para corregir los elementos que limitan el desarrollo del proceso, de este modo el sistema contempla la oportunidad de crear propuestas, las cuales parten desde las aportaciones y perspectivas de los colaboradores de la organización en conjunto con los resultados encontrados.

El sistema presentado puede recibir ajustes si se llegan a encontrar nuevas necesidades surgidas a partir de las expectativas del CRM, los resultados del proceso tienen un impacto directo en las decisiones de marketing del negocio y en consecuencia contribuirán al desempeño de la compañía. La visión mega de la organización está estrechamente ligada con los resultados que se esperan, ya que el producto de calidad que comercializa la compañía tiene requisiciones especiales tasadas en estándares internacionales de calidad, que van en el sentido de satisfacer las necesidades de los consumidores otorgando un producto sano que no daña la salud de las personas.

Por otra parte, la estrategia CRM requiere que la dirección de la organización y el departamento de marketing trabajen alineadas, con la convicción de que los resultados se verán reflejado a largo plazo, conforme se adquiera la experiencia de la iniciativa implantada, la cual llevará a hacer más eficiente el proceso. De igual manera, la empresa debe motivar a sus colaboradores y exponer periódicamente los avances alcanzados, reconociendo los esfuerzos realizados por el equipo de trabajo.

\section{REFERENCIAS}

Abad, P. (2009). Modelo CRM para un hotel de 4 estrellas. D.F., México: Instituto Politécnico Nacional: Escuela Superior de Turismo. Recuperado el 25 de Noviembre de 2014, de http:/ /tesis.bnct.ipn.mx:8080/dspace/bitstream/ 123456789/6887/1/MODELOCRM.pdf.

Amo, F. (2011). El cuadro de mando integral: balanced scorecard. Madrid, España: ESIC Editorial D.L. Recuperado el 9 de Diciembre de 2014, de http:/ /www.worldcat.org/title/cuadro-de-mando-integralbalanced-scorecard/oclc/796371927.

Arriagada, R. (2002). Diseño de un sistema de medición de desempeño para evaluar la gestión municipal: una propuesta metodológica. Santiago, Chile: CEPAL. Recuperado el 8 de diciembre de 2014, de http://www.worldcat.org/title/diseno-de-unsistema-de-medicion-de-desempeno-paraevaluar-la-gestion-municipal-una-propuestametodologica/oclc/51007532/viewport.

Bernárdez, M. L. (2009). Desempeño Humano. Bloomington, Indiana: AuthorHouse.

Cabanelas, L., \& Moreira, A. (2009). La gestión de las relaciones con clientes y la empresa de alta rentabilidad de Galicia. Revista de Estudios Regionales (84), 223-246. Recuperado el 3 de Diciembre de 2014, de http://redalyc.uaemex.mx/ src/inicio/ArtPdfRed.jsp?iCve=75511780008.

Garrido, A. (2008). La gestión de relaciones con clientes (CRM) como estrategia de negocio: Desarrollo de un modelo de éxito y análisis empírico en el sector hotelero español. Málaga, España: Universidad de Málaga: RiUMA. Recuperado el 20 de Noviembre de 2014, de http://riuma.uma.es/xmlui/ handle/10630/4606.

Guerra-López, I. (2007). Evaluación y Mejora Continua: Conceptos y Herramientas Para la Medición y Mejora del Desempeño. Bloomington, Ind: AuthorHouse.

Guijarro, M. (2009). Estudio de la literatura y modelos de negocio de la implantación de CRM- modelo cliente céntrico - como enfoque estratégico condicionante de la ventaja competitiva en la PYME: estudio empírico de la aplicación de un CRM - modelo cliente céntrico. Valencia, España: Universidad Politécnica de Valencia. Recuperado 
el 12 de Noviembre de 2014, de http://riunet.upv.es/ bits trea m/h andle/10251/7769/ tesisUPV3179.pdf?sequence $=11$.

Kaplan, R. S., \& Norton, D. P. (2004). Mapas estratégicos. Barcelona: Harvad Busines School Publishing Corporation.

Loor, K. (2013). Mejoramiento del Trabajo Autónomo le los Estudiantes de la Carrera Licenciatura en Inglés de la Universidad Estatal Península de Santa Elena a Través del Curso Reading and Writing I con la Modalidad Blended Learning 20122013.Experiencia Pedagógica. Universidad Estatal Península de Santa Elena, 30-36. Recuperado el 8 de Diciembre de 2014, de http://upse.edu.ec/ $\mathrm{rcpi} / \mathrm{images} / \mathrm{RV}$ _REVISTA_CPI_3/

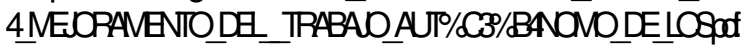

Muñoz, P. C., \& González, M. (2009). El diseño de materiales de aprendizaje multimedia y las nuevas competencias del docente en contextos teleformativos. Madrid: Bubok Publishing S.I. Recuperado el 9 de diciembre de 2014, de http:// www.worldcat.org/title/diseno-de-materiales-deaprendizaje-multimedia-y-las-nuevascompetencias-del-docente-en-contextosteleformativos/oclc/865826072.

Santana-Robles, F., \& Granillo-Macías, R. (2012). Identificación de atributos para la medición del desempeño del Sistema Producto Cebada del estado de Hidalgo, México. Científica, 16(1), 1123. Recuperado el 8 de Diciembre de 2014, de h t t p : / / w w w. red a I y c.org / articulo.oa?id=61424633002.

Valenzuela, L., \& Torres, E. (2008). Gestión empresarial orientada al valor del cliente como fuente de ventaja competitiva. Propuesta de un modelo explicativo. Estudios Gerenciales, 24(109), 65-86. Recuperado el 13 de Diciembre de 2014, de http:// redalyc.uaemex.mx/s rc/inicio/ ArtPdfRed.jsp?iCve=21211518003.

Vogel, M. (9 de diciembre de 2014). Como realizar un cuadro de Mando Integral. Recuperado el 16 de Noviembre de 2012, de http:// tablerodecomando.com/manuales/ Cuadro_de_Mando_Integral.pdf 


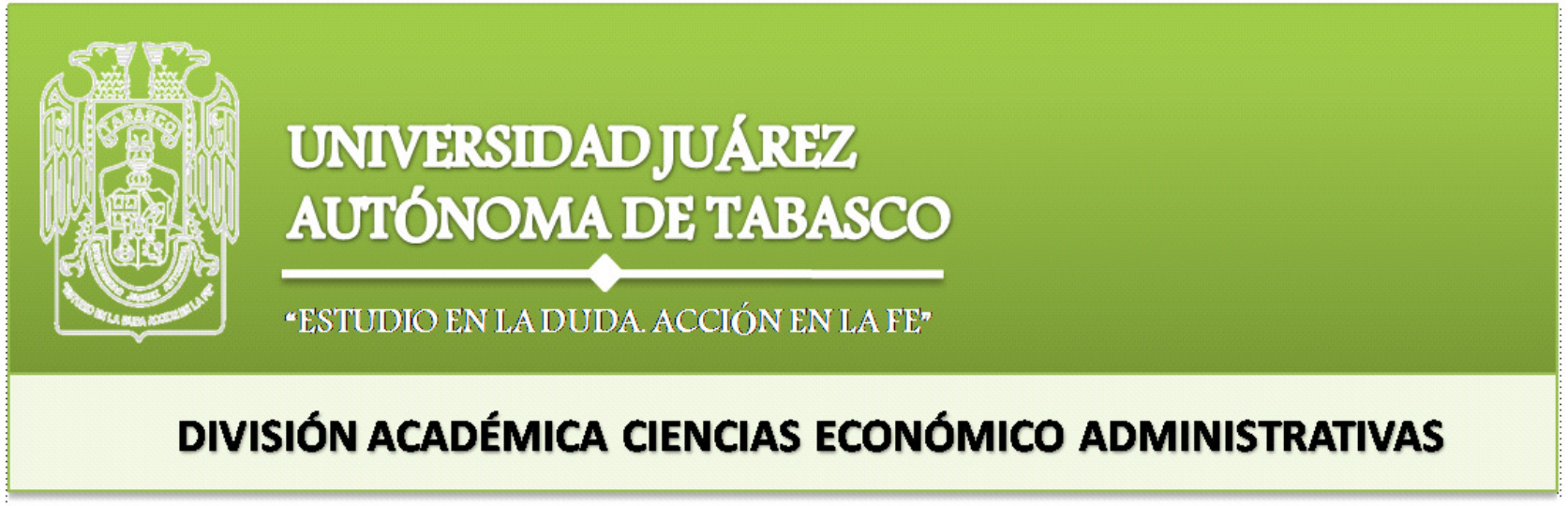

\section{DIPLOMADO}

LINEAMIENTOS Y FUNDAMENTOS METODOLÓGICOS PARA LA ELABORACIÓN DE TESIS EN LOS ESTUDIOS DE POSGRADOS

\section{Módulos:}

1. Delimitación del Tema de Investigación.

2. Desarrollo del Marco de Referencia: Teórico y Contextual

3. Metodología de la Investigación.

4. Análisis y presentación de los resultados

\section{Duración del diplomado: 160 horas}

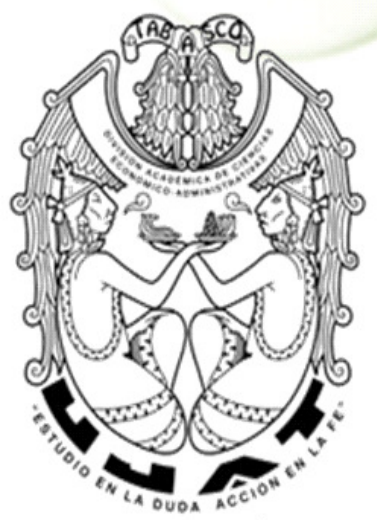

Área de educación contunua
Requisitos:

1. Haber concluido satisfactoriamente el Plan de Estudios de los estudios de Posgrados de la DACEA.

2. Presentar protocolo de investigación.

3. Constancia de terminación de estudios.

4. Constancia de comprensión de textos en ingles emitida por el CEI

\section{¡INSCRIPCIONES ABIERTAS!}

\section{INFORMES:}

EDIFICIODEEDUCACIÓNCONTINUA Y A DISTANCIA

M.A. WILLIAM BALDEMARLÓPEZRODRIGUEZ

TELEFONO: 3581567 EXT. 6219

Correo electrónico: educacioncontinua.dacea@ujat.mx 